\title{
The inconvenience of motherhood during a medical career
}

\author{
Andrea N. Simpson MD MSc, Maria C. Cusimano MD PhD, Nancy N. Baxter MD PhD
}

- Cite as: CMAJ 2021 September 20;193:E1465-6. doi: 10.1503/cmaj.211255

B alancing the competing demands of parenthood and a career in medicine is a challenge that many physicians and physicians-in-training experience. Delay of childbearing to attain specific career milestones may result in increased rates of infertility and adverse pregnancy outcomes among physicians. ${ }^{1}$ Conversely, having children earlier while in medical training is challenging and may influence a trainee's career decisions. Assumptions regarding how a physician's priorities will change when they bear children may result in fewer career opportunities and encouragement to pursue "familyfriendly" specialties. ${ }^{2}$ Yet, the onus is placed on women physicians to succeed in a profession that historically excluded women and has only recently acknowledged the importance of physician wellness and cultivating personal fulfillment. In spite of recent advances in the promotion of physician well-being, parenthood, especially motherhood, is seen as an inconvenience during medical training and beyond; in the medical profession, it seems there is really no "good" time to have children. Our profession is faced with research indicating that as many as 1 in 4 women physicians report a diagnosis of infertility. ${ }^{1}$ This should spark a call to action for systemic change in medicine, particularly as training pathways for physicians are being extended.

Women physicians commonly report delay of personal life decisions owing to career pursuits, ${ }^{3,4}$ and those who do pursue pregnancy during training have often reported maternal discrimination, resulting in negative career implications. ${ }^{5}$ Maternal discrimination refers to gender-based discrimination specifically based on motherhood status; it may be experienced as lack of support during pregnancy and postpartum, lack of accommodations for childcare challenges, disparaging remarks related to pregnancy and motherhood, and exclusion from career opportunities. ${ }^{5}$ Women medical students are also more likely than men to experience gender-based discouragement to specialize in surgery, based on assumed family aspirations. ${ }^{6}$ When these factors are considered, it is not surprising that many women physicians choose to delay pregnancy; however, many may experience a high personal cost, with increased rates of miscarriage, infertility and pregnancy complications associated with late maternal age..$^{1,7}$

Medical training often coincides with the optimal biological age for physicians to have children. Women physicians face difficult

\section{Key points}

- Childbearing during medical training and later in physicians' careers is still often seen as an inconvenience or burden, despite growing emphasis on physician well-being.

- Owing to stigma and systemic barriers, many physicians begin childbearing at a later age than their nonphysician counterparts, which may be associated with an increased risk of infertility, miscarriage and other pregnancy complications.

- Women physicians are delaying childbearing for longer, which may be related to extended training pathways.

- Interventions to reduce discrimination against childbearing physicians may include increasing the number of residency positions to ensure scheduling flexibility, integrating reproductive-health teaching for physicians into medical curricula early in training, and fostering allyship in the profession to reduce stigma.

decisions as to whether they will have children, when, and how many, while still pursuing their career goals. We recently mapped the trajectory of childbirth in women physicians in a populationbased study using data from Ontario, Canada ${ }^{8,9}$ and found that the culture of delay starts early and persists throughout postgraduate training. We found a significantly reduced rate of childbirth among women physicians compared with nonphysicians overall, and an accelerated rate of childbirth only at later reproductive ages, suggesting that women physicians appeared to defer pregnancy until after training, with specialists delaying pregnancy longer than family physicians.

As a profession, we need to think more ambitiously about how we can support physicians who wish to have children at any career stage. The provision of parental leave policies and increased financial support, parameters for overnight call during pregnancy for postgraduate trainees, and practical accommodations such as ensuring availability of lactation resources within hospitals have been substantial, positive system- and institutionlevel interventions. ${ }^{10}$ However, despite these changes, ${ }^{10}$ we found that the length of delay of pregnancy is increasing - women physicians born between 1985 and 1994 had a lower cumulative incidence of childbirth at comparable ages than those born 
between 1976 and $1984 .{ }^{8}$ Although extended training pathways or increased challenges in securing a stable career may be contributing factors, such findings also reflect underlying assumptions that drive maternal discrimination in medicine. Physicians going on pregnancy and parental leave often describe their time away as a "burden" - as do their peers - that increases the workload of others. ${ }^{5}$ Medical students and residents often cannot afford flexible childcare options. Women physicians report pervasive maternal discrimination, ${ }^{5}$ with fewer career opportunities after they have children. ${ }^{2}$

Physician reproductive health deserves more education, particularly early in medical training, to ensure physicians can make informed decisions regarding their family planning. Although fertility preservation through elective egg freezing is often presented as a way to "work around" the demands of medical training, it is not a failsafe and certainly not an ideal solution to the larger issue of delayed pregnancy; it should not be the only option. Our training environments need to change such that every specialty is supportive of people who wish to become parents.

Although we have focused primarily on issues pertaining to motherhood in medicine, balancing family and a career in medicine should not be thought of solely as a woman's issue - the perspectives of men and gender-diverse physicians are important and under-researched. To promote allyship, it is important to engage physicians of all genders, particularly those in leadership positions, to view parental leave as key to the health of physicians and their children, rather than regarding it as a burden on one's colleagues. On a broader scale, increased funding for residency positions - to ensure an adequate number of trainees to cover service needs related to parental leave - may foster acceptance of pregnancy and parental leave by fellow trainees and improve scheduling flexibility. The repercussions of discrimination against physicians who would bear children are substan- tial, extending to some women physicians changing practice locations owing to toxic work environments, or leaving medicine altogether. ${ }^{5}$ An environment that results in a physician leaving the practice of medicine after investing years of training in the profession is an avoidable tragedy for the individual, the medical community and affected patients. Fostering systemic positive change in medicine to support physicians who have children at any training stage will promote wellness and career satisfaction in the long term.

\section{References}

1. Stentz NC, Griffith KA, Perkins E, et al. Fertility and childbearing among American female physicians. J Womens Health (Larchmt) 2016;25:1059-65.

2. Adesoye T, Mangurian C, Choo EK, et al.; Physician Moms Group Study Group. Perceived discrimination experienced by physician mothers and desired workplace changes: a cross-sectional survey. JAMA Intern Med 2017;177:1033-6.

3. Bering J, Pflibsen L, Eno C, et al. Deferred personal life decisions of women physicians. J Womens Health (Larchmt) 2018;27:584-9.

4. Nasab S, Shah JS, Nurudeen K, et al. Physicians' attitudes towards using elective oocyte cryopreservation to accommodate the demands of their career. J Assist Reprod Genet 2019;36:1935-47.

5. Halley MC, Rustagi AS, Torres JS, et al. Physician mothers' experience of workplace discrimination: a qualitative analysis. BMJ 2018;363:k4926.

6. Giantini Larsen AM, Pories S, Parangi S, et al. Barriers to pursuing a career in surgery: an institutional survey of Harvard Medical School students. Ann Surg 2021;273:1120-6.

7. Rangel EL, Castillo-Angeles M, Easter SR, et al. Incidence of infertility and pregnancy complications in US female surgeons. JAMA Surg 2021 July 28 [Epub ahead of print]. doi: 10.1001/jamasurg.2021.3301.

8. Cusimano MC, Baxter NN, Sutradhar R, et al. Delay of pregnancy among physicians vs nonphysicians. JAMA Intern Med 2021;181:905-12.

9. Cusimano MCBN, Sutradhar R, Ray J, et al. Reproductive patterns, pregnancy outcomes and parental leave practices of women physicians in Ontario, Canada: the Dr Mom Cohort Study protocol. BMJ Open 2020;10:e041281.

10. Pregnancy and parental leave. Toronto: Professional Association of Residents of Ontario (PARO). Available: https://myparo.ca/your-contract/\#pregnancy -and-parental-leave (accessed 2021 June 16).
Competing interests: None declared.

This article has been peer reviewed.

Affiliations: Department of Obstetrics and Gynaecology (Simpson, Cusimano), University of Toronto; Li Ka Shing Knowledge Institute (Simpson, Baxter), St. Michael's Hospital; Department of Obstetrics and Gynecology (Simpson, Cusimano, Baxter), St. Michael's Hospital/Unity Health Toronto; ICES Central (Simpson), Toronto, Ont.; Melbourne School of Population \& Global Health (Baxter), University of Melbourne, Melbourne, Victoria, Australia
Contributors: All of the authors contributed to the conception and design of the work. Andrea Simpson drafted the manuscript. All of the authors revised it critically for important intellectual content, gave final approval of the version to be published and agreed to be accountable for all aspects of the work.

Funding: The referenced studies by the authors 8,9 were conducted with grant funding from Physicians' Services Incorporated Foundation.
Content licence: This is an Open Access article distributed in accordance with the terms of the Creative Commons Attribution (CC BY-NC-ND 4.0) licence, which permits use, distribution and reproduction in any medium, provided that the original publication is properly cited, the use is noncommercial (i.e., research or educational use), and no modifications or adaptations are made. See: https://creativecommons. org/licenses/by-nc-nd/4.0/

Correspondence to: Andrea Simpson, andrea.simpson@unityhealth.to 